\title{
Einstein's Conflicting Heuristics: The Discovery of General Relativity
}

\author{
John D. Norton 1 \\ Department of History and Philosophy of Science \\ University of Pittsburgh \\ Pittsburgh PA USA 15222 \\ http://www.pitt.edu/ jdnorton
}

\section{Introduction}

What were the heuristics that guided Einstein to his completed general theory of relativity of 1915? There can be no simple answer. The completion of the theory came only after eight years of exhausting labor. In them, Einstein, at the height of his creative powers, grappled with problems so profound that they nearly defeated him. Nonetheless, Einstein himself provided an appealing and simple narrative of his discovery. He was guided, he assured us, by a few simple but powerful physical principles and thought experiments. These same heuristics then became the basis of Einstein's later account of the logical foundations of general relativity.

In narrowing his focus to these few heuristics, Einstein purged his account of nearly all the complications and false steps that later historical work has revealed. It obscures the fact that there is a great distance between the lofty generalities of Einstein's principles and the messy details of the final theory. These principles could not by themselves have led Einstein to the final theory. Worse, as will be recounted in Section 2 below, most of the heuristics of this narrative turn out not to be vindicated by Einstein's final theory and may even fail to be sustainable as independent ideas. They provided at best an unreliable guide and a dubious logical foundation for the theory.

So we must ask again, what were the heuristics that guided Einstein? Something more must have helped Einstein arrive at his final success. My contention in this paper is that beneath this first tier of heuristics lies a second tier of heuristics. They do not lend themselves to arresting

${ }^{1}$ I thank Michel Janssen for helpful discussion in general and especially relating to Section 13. 
statements of a grand vision. Rather they are the practical lessons that a theorist like Einstein learns as he returns day after day to the mundane work of theory building. Whatever else may happen, Einstein's new theory must conserve energy and mesh with Newton's old theory of gravity. Getting all these details to work is not easy. A theorist can readily be led into blind alleys. The theorist must learn the tricks that avoid the traps. Once they are learned, it is all too easy to omit them from the celebratory recollections. However it is the accretion of these lesser heuristics that proves as important to the final discovery. Without them, the final result could not be achieved.

This paper traces the trace the fortunes of just one of Einstein's first-tier heuristics, the principle of equivalence. It did guide Einstein's thinking. However the principle was defeasible. We shall see that it was diluted in 1912 and all but discarded in 1913 when a second tier heuristic, the requirement of conservation of energy and momentum, led to gravitational field equations that contradicted it. This second tier heuristic was beyond challenge. It persisted and powerfully circumscribed Einstein's continuing analysis up to the completion of his theory in 1915.

In the following, Sections 2 to 6 describe the origin of the principle in Einstein's earliest reflections on gravitation and acceleration and traces how it guided Einstein to a novel theory of static gravitational fields in 1912. Sections 7 to 11 recall how Einstein found that the resulting theory conflicted with a second tier heuristic, the conservation of momentum. Reluctantly, Einstein was compelled to modify the theory's single field equation to one that compromised his principle of equivalence. The principle could now only hold, as he put it, for infinitely small fields.

The following year, as recounted in Section 12, Einstein and his mathematician friend Marcel Grossmann devised the "Entwurf" theory. It differed from general relativity only in employing gravitational field equations of limited covariance. Conservation of momentum had, in 1912, forced a quite specific field equation on Einstein. He now turned that experience to his advantage. Einstein went to pains to explain in elementary terms that the conservation requirement provides a general method for arriving at unique field equations. He then used it with the conservation of energy and momentum to identify the gravitational field equations of "Entwurf" theory. What Einstein did not then acknowledge clearly, as Section 13 recalls, was that his original principle of equivalence now failed completely with this choice of field 
equations. Whatever the merits of this first tier heuristic, its role in theory formation was quite displaced by the second tier heuristic of the conservation of energy and momentum.

This second tier heuristic enjoyed a brief moment of prominence when it was highlighted in Einstein and Grossmann's (1913) "Entwurf” paper as the foundation of the method used to derive the theory's gravitational field equations. Section 14 recounts how the heuristic became less visible but continued to exercise a controlling role in Einstein's subsequent theorizing. It governed Einstein's analysis of the limited covariance of the "Entwurf" theory and persists in modern accounts of general relativity in providing the fastest route to Einstein's celebrated gravitational field equations of 1915 .

Section 15 and the concluding Section 16 review the tension between the conception and application of the heuristics of the two tiers. Einstein's recounting accords the first tier heuristic, the principle of equivalence, primary foundational importance. Yet it was defeasible in his actual theorizing when it conflicted with a second tier heuristic, the conservation of energy and momentum. Section 15 also reviews briefly a related episode of heuristics in tension that was explored in some detail by Janssen and Renn (2007). It concerns Einstein's November 1915 return to generally covariant field equations. Two appendices contain background calculations.

\section{Einstein's Principles}

Einstein completed his general theory of relativity in November 1915. The triumph came to an exhausted and exhilarated Einstein after eight years of labor on the problem of relativity and gravitation. It was a distinctive achievement, quite unlike so many other discoveries in physics. In these other cases, novel empirical results were key. The final theory lay hidden in them in encoded form. Success came when someone figured out how to read the code. The nineteenth century accumulated a wealth of empirical results on electricity and magnetism. They were summarized in the Maxwell-Lorentz electrodynamics that the young Einstein studied so eagerly. Encoded in them he found the Lorentz transformation and with it the special theory of relativity of $1905 .^{2}$ In the same year, Einstein found his revolutionary light quantum hypothesis.

\footnotetext{
2 See Norton (2004) for an account of Einstein's investigations.
} 
It was encoded, he realized, in the recently-measured thermodynamic properties of heat radiation. ${ }^{3}$

The discovery of general relativity was quite unlike these. There was some empirical guidance. Perhaps the most significant empirical result guiding Einstein was an old one. It was a commonplace since the work of Galileo and Newton in seventeenth century that all bodies fall under gravity with the same acceleration, independently of their masses. Aside from this result, on his own account, the heuristics that guided Einstein were more ethereal and philosophical in character. At their center was what Einstein labeled "an epistemological defect" in prior theories of both classical mechanics and special relativity. ${ }^{4}$ These theories were defective in positing inertial frames of reference since their disposition was fixed absolutely without relation to the contents of space and time. The associated, preferred inertial motions were absolute in a sense Einstein (1923, p. 61) found objectionable: "independent in its physical properties, having a physical effect, but not itself influenced by physical conditions." To eliminate this defect, Einstein proposed that the principle of relativity had to be extended from the relativity of inertial motion of his 1905 special theory of relativity to include accelerated motion as well.

The need for this extension was grounded further in an idea that Einstein attributed ${ }^{5}$ to Ernst Mach: that the inertia of bodies is due to an interaction with the other masses of the universe. This, he labeled "Mach's principle." According to it, the distribution of matter in space determines completely the disposition of the inertial frames of reference. Finally there was what Einstein called 6 the "happiest thought of [his] life," the principle of equivalence. It asserted the equivalence of uniform acceleration in gravitation free space and a homogeneous gravitational field. This principle, Einstein was already able to boast at the outset in (Einstein, 1907, p. 454), "extends the principle of relativity to uniformly accelerated translational motion of the reference system.” It was, he felt, a promising first step.

\footnotetext{
3 See Norton (2006) for an account of this encoding.

4 “ein erkenntnistheoretischer Mangel” Einstein (1916, p. 771).

5 There is some question over whether Einstein's attribution to Mach was correct. See Norton (1995).

6 Einstein (1920a, p. 265).
} 
These heuristics are widely celebrated. They are almost as well-known as the iconic photographs of Einstein, the disheveled genius and iconoclast. Their popularity is driven by their vividness and simplicity. They lend themselves to memorable thought experiments. The principle of equivalence is routinely expressed through the parable of an observer trapped in a box or an elevator. The box is accelerated in gravitation free space; or, in later variants, the box is in free fall in a gravitational field. Mach's principle is routinely related as an answer to Newton's own bucket thought experiment. Newton had proposed in his Principia that the concavity in the surface of the water in a rotating bucket arises from acceleration with respect to absolute space. Mach's principle asserts that, instead, the concavity arose from the water's acceleration with respect to all the other masses of the universe.

These heuristics promise an easy pathway to understanding a theory that, reputedly, is so abstruse that few can properly understand it. ${ }^{7}$ Everyone who has driven in a car understands viscerally how acceleration produces inertial forces. These, we are told on Einstein's authority, are really gravitational forces; and they arise precisely because you are accelerating in relation to all the other masses of the universe. Appreciate that and an understanding the general relativity of all motion is almost within your grasp. It seems so easy.

Einstein's autobiographical statements leave no doubt of the importance of these heuristics in Einstein's process of discovery. However there is a troubling aspect to them. They depend heavily on judgments of how physical theories have to be, independent of experience. Such efforts are rarely successful. Time and again today's experience or more careful thought has overturned yesterday's theoretical imperatives. So it is with Einstein's heuristics. Many do not survive scrutiny. 8

There is no deeper principle of nature that requires us to eschew something that has (in Einstein's words) "a physical effect, but [is] not itself influenced by physical conditions." Whether inertial frames of reference are as special relativity dictates is a matter to be decided empirically and not by a priori stipulation. Contrary to Einstein's earlier hopes, the Machian principle turned out not to be implemented in the final general theory of relativity. Einstein

\footnotetext{
7 An anonymous preface to Lorentz (1920, p. 5) Begins "Whether it is true or not that more than twelve persons in all the world are able to understand Einstein's Theory, ..."

${ }^{8}$ For a synoptic survey of the problems in Einstein's heuristics, see Norton (1993).
} 
eventually abandoned the principle as incompatible with a thorough-going field theoretic approach. The generalization of the principle of relativity to accelerated motion was implemented by Einstein as a demand that his new theory be expressible in arbitrarily chosen spacetime coordinate systems. Kretschmann quite correctly objected in 1917 that this requirement was all but vacuous. It was more a challenge to the ingenuity of theorists in the way they wrote their equations. Finally Einstein's original formulation of the principle of equivalence almost immediately disappeared from the literature. In its place came a proliferation of variant forms ("weak," "strong," "Einstein") that differed from Einstein's in both fundamental conception and content.

Troubled as these heuristics are, there is no doubt of their importance in Einstein's mind while he worked on the problem of relativity and gravitation. If they were his only guides, then it would be somewhat more than extraordinary that his deliberations should produce such a remarkable result, the general theory of relativity. There were, as we shall now see, many more guides. They were buried in details that did not lend themselves to popular exposition.

The attempt in this paper to understand how Einstein succeeded nonetheless proceeds in the spirit of Janssen (2014), who addresses the same question. In his concluding Section 6, entitled “Post Mortem: How Einstein’s Physics Kept his Philosophy in Check,” Janssen attributes Einstein's success to three factors:

First, Einstein did not just want to eliminate absolute motion, he also wanted to reconcile some fundamental insights about gravity with the results of special relativity and integrate them in a new broader framework. Second, when these efforts led him to the introduction of the metric field, he carefully modeled its theory on the successful theory of the electromagnetic field of Maxwell and Lorentz. Third, whenever his philosophical agenda clashed with sound physical principles, Einstein jettisoned parts of the former instead of compromising the latter. The analysis of this paper illustrates the third of these factors. Einstein's principle of equivalence belongs in what Janssen calls the "philosophical agenda." It is here a defeasible, first tier heuristic. Conservation of energy and momentum is one of Janssen's "sound physical principles." It belongs in the second tier of heuristics that cannot be compromised. 


\section{Einstein's 1907 Heuristic}

The project began in 1907 when Einstein was commissioned to write a review article on the "principle of relativity," this being the term that delineates what we would now call the special theory of relativity. The resulting review article, Einstein (1907), showed how existing branches of physics could accommodate or be accommodated to Einstein's new theory of space and time. Only one area of physics proved troublesome: gravitation. In Section V, Einstein embarked on a speculative new approach to gravity that might at the same time afford an extension of the principle of relativity to accelerated motion.

The heuristic device that guided Einstein was labeled merely as an "assumption" (Annahme). In what we must presume was the space of Newtonian mechanics, he considered a reference system $\Sigma_{1}$ uniformly accelerated in a fixed direction; and a second inertial reference system $\Sigma_{2}$ in which there is a homogeneous gravitational field. He supposed further that the acceleration of $\Sigma_{1}$ matched the acceleration of fall of free bodies in $\Sigma_{2}$, so that the motions of free bodies would be the same in both systems. Einstein's assumption was that this sameness was to be generalized to all physical processes. We must presume a tacit extension to relativistic contexts. He wrote (p. 454):

We have therefore in the present state of our experience no basis for the assumption that the systems $\Sigma_{1}$ and $\Sigma_{2}$ differ from one another in any respect. Hence we want to assume in the following the complete physical equivalence of a gravitational field and the corresponding acceleration of the reference system.

This assumption extends the principle of relativity to the case of uniformly accelerated translational motion of the reference system....

Modern readers will immediately recognize this as Einstein's first statement of the principle of equivalence. They may however be puzzled by the restriction of equivalence to the special case of a homogeneous gravitation field and uniform acceleration. Standard modern statements of the principle of equivalence are more general. They commonly assert that a gravitational field can always be transformed away, at least locally, by adopting an appropriate acceleration of the reference system.

That this more general version of the principle was not Einstein's has been recounted in Norton (1985). We need not rehearse here Einstein's objections to the generalized principle. The 
important point is to recognize that the principle was, for Einstein in 1907, not yet a permanent axiom of some well-articulated theory. That may still come. In 1907, the primary interest of the assumption for Einstein was as a heuristic guide in the generation of a new theory of gravity, whose general outlines were only dimly visible to Einstein in 1907. Einstein stated clearly his heuristic purpose in the continuation of the passage quoted above

... The heuristic value of the assumption lies in the fact that a homogeneous gravitational field may be replaced by a uniformly accelerated reference system.

The latter case is accessible to theoretical treatment to a certain degree.

There was then, in Einstein's view, an urgent need for such a heuristic. For Einstein had tried an obvious accommodation of gravity to special relativity, that is, the construction of simple, Lorentz covariant theories of gravity. Einstein (1933, pp. 286-87) recalled the problem he discovered:

These investigations, however, led to a result which raised my strong suspicions. According to classical mechanics, the vertical acceleration of a body in the vertical gravitational field is independent of the horizontal component of its velocity. Hence in such a gravitational field the vertical acceleration of a mechanical system or of its center of gravity works out independently of its internal kinetic energy. But in the theory I advanced, the acceleration of a falling body was not independent of its horizontal velocity or the internal energy of the system.

This did not fit in with the old experimental fact that all bodies have the same acceleration in a gravitational field...

In short, Einstein had failed to find a relativized theory of gravity in which bodies fall vertically with equal acceleration, independently of their horizontal motion. ${ }^{9}$ How could Einstein proceed? The assumption of 1907 - the principle of equivalence - provided a way. It delivered to Einstein a single instance of a gravitational field with just the independence property needed. To proceed, all Einstein needed to do was to catalog the properties of this one special case of a relativized gravitational field and then judiciously generalize them to recover a full theory.

\footnotetext{
${ }^{9}$ For an attempted reconstruction of Einstein’s explorations, see Norton $(1992, \S 3)$.
} 


\section{Einstein 1907-1912 Theory of Static Gravitational Fields}

This project of generalization became the substance of those parts of the ensuing five years that Einstein devoted to gravitation. The 1907 review article already contained some now familiar results. The speed of light and the ticking of clocks would be slowed in a homogeneous gravitational field. This speed played the role of a gravitational potential. These results, now generalized to the inhomogeneous static gravitational field of the sun, yielded a prediction of a slight red shift in light emitted by the sun. Einstein returned to work on the theory in 1911, when he realized that another effect in it was open to observational test. According to the theory (Einstein, 1911), a beam of light is bent by a gravitational field. The bending should be detectible in a displacement of apparent star positions in the sky in the vicinity of the sun.

While this 1911 analysis is widely know through its inclusion in the ubiquitous Dover reprint The Principle of Relativity, the fullest expression of the project of generalization came in a lesser-known pair of papers the following year (Einstein 1912a, 1912b). These papers contained a full theory of certain static gravitational fields. The theory provided equations of motion for bodies in free fall, a field equation for the variable speed of light and versions of electrodynamics and thermodynamics, suitably modified to accommodate the novelty of a variable speed of light.

The starting point of the paper is a transformation from the familiar reference system $\Sigma$ of Einstein's 1905 special theory of relativity, represented by coordinates of space and time $(\xi, \eta, \zeta$, $\tau)$, to a unidirectionally, uniformly accelerated frame of reference $K$, represented by the coordinates of space and time $(x, y, z, t)$. Einstein's analysis is cumbersome. He does not develop the full transformation equations, although (as we are about to see), they are quite simple. In a labored development proceeding over many pages, he recovers only an approximation of the general transformation equations for small $t$. Einstein's generalizations proceed from them.

Here I will not recapitulate these details. They would provide no special illumination for the issues to be raised. Instead I will summarize them using a more perspicacious formalism that Einstein himself shortly recognized. In a last minute correction to the proofs of Einstein (1912b, p. 458), Einstein found his equations of motion is recovered most simply from an action principle. The following year Einstein and Grossmann (1913, p.7) revealed that this action 
principle is the equation of geodesic motion in a spacetime whose structure is no longer Minkowskian.

\section{The Gravitational Field of Uniform Acceleration}

The equations relating the unaccelerated and uniformly accelerated frame $\Sigma$ and $K$ were given later in many places, including Einstein and Rosen (1935, p. 74): 10

$$
\tau=\left(c_{0} / a+x\right) \sinh (a t) \quad\left(\xi+c_{0} / a\right)=\left(x+c_{0} / a\right) \cosh (a t) \quad \eta=y \quad \zeta=\mathrm{z}
$$

The acceleration is uniform translational acceleration in the $\xi, x$ direction; $a$ is a constant acceleration parameter and $c_{0}$ a constant. While the original coordinates of $\Sigma$ cover the whole of the spacetime, those of $K$ cover only a wedge delimited by null surfaces $\tau=\left(\xi+c_{0} / a\right)$ and $\tau=-\left(\xi+c_{0} / a\right)$. The $x$ coordinate can only take values greater than $-c_{0} / a$, for the coordinates are singular at $x=-c_{0} / a$, where all the hypersurfaces of constant $t$ intersect. ${ }^{11}$ When $t$ is small, the hyperbolic functions in (1) are well-approximated as $\sinh (a t) \approx a t$ and $\cosh (a \mathrm{t}) \approx 1+a^{2} t^{2} / 2$. Then the exact transformation equations (1) are well-approximated by the small $t$ expressions Einstein derived in Einstein (1912, p. 359):

$$
\tau=\left(c_{0}+\mathrm{a} x\right) t \quad \xi=x+\left(c_{0}+\mathrm{a} x\right) a t^{2} / 2 \quad \eta=y \quad \zeta=\mathrm{z}
$$

Under the transformation (1), using the perspectives Einstein would develop the following year, the Minkowskian expression for the invariant line element 12

$$
d s^{2}=d \tau^{2}-d \xi^{2}-d \eta^{2}-d \zeta^{2}
$$

10 The notation is adapted to Einstein's (1912a) usage. The Einstein and Rosen version was the slightly simpler case in which $c_{0}=0$.

${ }^{11}$ In 1912, since he worked only with a small $t$ approximation (2), Einstein may not have realized that the coordinates $(x, y, z, t)$ he introduced have a singularity at $x=-c_{0} / a$. Einstein and Rosen (1935) later suggest that one can conceive the "field-producing mass" as located at this singularity, although they seek to eliminate the singularity. In (1912a, p. 356, footnote), however, Einstein wrote: "The masses that produce this field should be conceived as at infinity."

12 The notation is adapted to Einstein's (1912a) usage. The Einstein and Rosen version was the slightly simpler case in which $c_{0}=0$. 
becomes

$$
d s^{2}=\left(c_{0}+a x\right)^{2} d t^{2}-d x^{2}-d y^{2}-d z^{2}
$$

While the transition from expression (3) to (4) has merely redistributed the coordinates assigned to events, Einstein used the principle of equivalence to conclude that a homogeneous gravitational field now manifests in the new frame of reference $K(x, y, z, t)$. We can read directly from the line element (4) the same properties that Einstein inferred for this field, but with greater effort on his part.

Einstein defined the speed of light $c$ in terms of the new coordinates as

$$
c^{2}=(d x / d t)^{2}+(d y / d t)^{2}+(d z / d t)^{2}
$$

where $(x(t), y(t), z(t))$ is the trajectory of a light pulse. We read immediately from (4) that this speed of light $c$ varies linearly with $x$ in the direction of the gravitational field 13

$$
c(x, y, z)=c_{0}+a x
$$

Hence it can represent the gravitational potential.

We also read from (4) that the hypersurfaces of constant $t$ are ordinary Euclidean spaces and that their coordinates $(x, y, z)$ are Cartesian coordinates with the familiar metrical significance. The same is no longer true of the time coordinate $t$. It can no longer be measured directly by clocks. Rather times elapsed on a clock at rest in the frame must be rescaled by a position dependent factor $\left(c_{0}+a x\right)$ if the corresponding time coordinate $t$ differences are sought.

The equations of motion of bodies in free fall in this homogenous field are recovered by seeking the geodesics of the spacetime, that is, those trajectories for which $\int d s$ is extremal. A short and standard calculation of the Euler-Lagrange equations yields

$$
\begin{gathered}
\frac{d}{d t}\left(\frac{\dot{x} /\left(c_{0}+a x\right)}{\sqrt{1-q^{2} /\left(c_{0}+a x\right)^{2}}}\right)=\frac{-a}{\sqrt{1-q^{2} /\left(c_{0}+a x\right)^{2}}} \\
\frac{d}{d t}\left(\frac{\dot{y} /\left(c_{0}+a x\right)}{\sqrt{1-q^{2} /\left(c_{0}+a x\right)^{2}}}\right)=\frac{d}{d t}\left(\frac{\dot{z} /\left(c_{0}+a x\right)}{\sqrt{1-q^{2} /\left(c_{0}+a x\right)^{2}}}\right)=0
\end{gathered}
$$

where $q^{2}=\dot{x}^{2}+\dot{y}^{2}+\dot{z}^{2}$ and the overhead dot denotes differentiation with respect to $t, \dot{x}=d x / d t$, etc.

13 The other case of $c(x, y, z)=-\left(c_{0}+a x\right)$ is not mentioned by Einstein. 


\section{Einstein Generalizes Naturally}

With these results in hand for the special case of a homogeneous gravitational field, Einstein could now proceed with his project of generalization. The generalizations he introduced were obvious and natural. ${ }^{14}$

First, the line element (4) is replaced by the more general

$$
d s^{2}=c^{2}(x, y, z) d t^{2}-d x^{2}-d y^{2}-d z^{2}
$$

where $c$ can now vary more generally as a function of the spatial coordinates. This variable speed of light $c$ still serves as the single gravitational potential and the spatial hypersurfaces of constant time coordinate $t$ remain Euclidean. This new structure represents a more general case of time independent gravitational fields. Einstein recognized explicitly (1912a, p.356), however, that it was not the most general case. He noted that the field produced by a rotation of the reference frame would yield a non-Euclidean geometry. For the Lorentz contraction would act differentially on rods oriented parallel or transverse to the direction of rotation. That meant that the ratio of the circumference of a suitably placed circle to its diameter would no longer be the Euclidean value of $\pi$, when both are measured by rods at rest in the rotating reference frame.

In the generalization, the gravitational field strength is the negative gradient of the speed of light, $(-\partial c / \partial x,-\partial c / \partial y,-\partial c / \partial z)$. The equations of motion of a body in free fall in a homogeneous gravitational field (7) are naturally generalized to

$$
\begin{gathered}
\frac{d}{d t}\left(\frac{\dot{x} / c}{\sqrt{1-q^{2} / c^{2}}}\right)=\frac{-\partial c / \partial x}{\sqrt{1-q^{2} / c^{2}}} \\
\frac{d}{d t}\left(\frac{\dot{y} / c}{\sqrt{1-q^{2} / c^{2}}}\right)=\frac{-\partial c / \partial y}{\sqrt{1-q^{2} / c^{2}}} \quad \frac{d}{d t}\left(\frac{\dot{z} / c}{\sqrt{1-q^{2} / c^{2}}}\right)=\frac{-\partial c / \partial z}{\sqrt{1-q^{2} / c^{2}}}
\end{gathered}
$$

14 This project may seem familiar since it is the first instance of what becomes the gauge argument routinely used to introduction interacting fields in particle physics. One starts with a flat connection, the case of no interaction. It is re-coordinatized (or its gauge changed) so that the new description mimics an interaction, while none is actually present. The new description is generalized to returns equations governing a non-trivial interaction. 
These equations coincide with the geodesics of the line element (8). They can also be recovered directly by solving the Euler-Lagrange equations using this more general line element (8).

Finally, Einstein sought a more general equation governing the speed of light $c$. The linear dependence of $c$ on $x$ in (6) is easily seen to be a solution of the Laplace equation for $c$ :

$$
\Delta c=\left(\frac{\partial^{2}}{\partial x^{2}}+\frac{\partial^{2}}{\partial y^{2}}+\frac{\partial^{2}}{\partial z^{2}}\right) c=0
$$

In turn, it is naturally generalized to:

$$
\Delta c=\left(\frac{\partial^{2}}{\partial x^{2}}+\frac{\partial^{2}}{\partial y^{2}}+\frac{\partial^{2}}{\partial z^{2}}\right) c=k c \sigma
$$

for $k$ a constant and $\sigma$ the matter density. This field equation is the obvious analog of Poisson's equation for the Newtonian gravitational potential $\varphi$

$$
\Delta \varphi=\left(\frac{\partial^{2}}{\partial x^{2}}+\frac{\partial^{2}}{\partial y^{2}}+\frac{\partial^{2}}{\partial z^{2}}\right) \varphi=4 \pi k \rho
$$

for mass density $\rho$ (in the form given in Einstein and Grossmann, 1913, p. 11).

The principal difference in form between (11) and (12) is that the first has a source term $k c \sigma$ that is linear in the potential $c$, whereas the source term of the second, $4 \pi k \rho$, has no corresponding term in $\varphi$. This difference reflects a difference in gauge freedoms in the two quantities $c$ and $\varphi$. The speed of light $c$ is undetermined up to a multiplicative factor $M$, reflecting our freedom to choose measuring units for distances and times. Thus, if $c$ is a solution of (11) for some $\sigma$, then so is $c^{\prime}=c M$. The Newtonian potential $\varphi$, however, is undetermined up to an additive factor A. Thus, if $\varphi$ is a solution of (12) for some $\rho$, then so is $\varphi^{\prime}=\varphi+A$.

This difference in gauge freedoms in the two cases may now seem innocuous. It will shortly prove to be a cause of considerable trouble.

\section{A Hidden Peril}

The generalizations of the last section are small and modest. They would be, it seems, just a small and secure step towards the most general theory. However as Einstein would shortly discover, these generalizations were far from innocent. The danger lay precisely in their apparent modesty, so that one would not readily think to challenge them. 
Buried in the generalizations were two, specific problems. The first was the idea that space would remain Euclidean in the case of more general static gravitational fields. This proves almost never to be the case. Take one of the simplest cases: the Schwarzschild spacetime, the exterior gravitational field of a rotationally symmetric, uncharged, non-rotating body of mass $m$. Its line element is

$$
d s^{2}=\left(1-\frac{2 G m}{r}\right) d t^{2}-\frac{d r^{2}}{\left(1-\frac{2 G m}{r}\right)}-r^{2}\left(d \theta^{2}+\sin ^{2} \theta d \phi^{2}\right)
$$

The constant $G$ is the Newtonian universal constant of gravitation and $(r, \theta, \phi)$ are spherical coordinates of space. The failure of Euclidean geometry for the spatial hypersurfaces of constant $t$ arises through the division of $d r^{2}$ by the factor (1-2Gm/r). For, without it, the spatial line element is the Euclidean $d r^{2}+r^{2}\left(d \theta^{2}+\sin ^{2} \theta d \phi^{2}\right)$.

The trouble with Einstein's 1912 assumption of spatial flatness is that it is incompatible with his final field equations of November 1915. As John Stachel (1989) first pointed out, as long as Einstein expected fields like (8) to satisfy his field equations, he is precluding the source free field equations of the vanishing of the Ricci tensor, $R_{\mu \nu}=0.15$ When Einstein adopted the mathematical framework of general relativity in his joint work with Grossmann (1913), notoriously, Einstein considered and rejected generally covariant gravitational field equations employing the Ricci tensor. This misstep marked the beginning of years of painful drifting, while Einstein sought to reconcile himself with a misshapen theory. Those years brought Einstein's formulation of his "hole argument." It sought to establish that generally covariant gravitational field equations would not be physically interesting. The assumption of spatial flatness supported his earlier prediction of only a "half deflection" in a beam of starlight grazing the sun. Einstein (1915b) found this error only at the last moment in November 1915, when his celebrated computation of Mercury's anomalous motion depended on the failure of Euclidean geometry in the vicinity of the sun.

15 The precise result is shown below in Appendix A. If the metric tensor is restricted to the form (36), then the vanishing of the Ricci tensor permits the $g_{00}=c^{2}(x, y, z)$ to vary at most linearly with the coordinates $x, y$ and $z$ as in (42). 
This one mistaken assumption was not the sole source of these years of misery for Einstein. However it was their starting point. I need only here reaffirm the profound and extended misery this assumption visited upon Einstein. For this episode has been the subject of very extensive historical investigations elsewhere, to which the reader is now directed. See Stachel (1989), Norton (1984), and for a synoptic work by Michel Janssen, John D. Norton, Jürgen Renn, Tilman Sauer and John Stachel that significantly develops these earlier papers, see Renn (2007).

\section{A Second Hidden Peril Identified}

While this last peril lingered on unnoticed for several years, there was a second peril that Einstein identified almost immediately. Einstein's first paper of 1912 (Einstein 1912a) had been submitted to Annalen der Physik on February 26, 2012. Before its printing was finalized, Einstein found to his dismay that the natural and obvious field equations (10) and (11) could not be exactly correct. He managed to append a footnote (p. 360) to them that alerted readers to the problem:

In a work to follow shortly, it will be shown that the equations [(10)] and [(11)] still cannot be exactly correct. They will be used provisionally in this work.

The work promised, Einstein (1912b), was submitted on March 23, 2012, to the journal, just under a month after the first paper was submitted. It dealt first with routine matters required by the new theory of gravity. Einstein showed how the theory required small adjustments to electrodynamics and thermodynamics. Section 4 of the paper then revealed the concern with the field equation.

Einstein considered a distribution of matter, momentarily at rest, where the gravitational potential $c$ produced by the matter approaches a constant potential at spatial infinity. The different parts of the matter distribution act gravitationally on one another. Gravitational collapse is prevented by attaching the masses to a rigid, massless frame. It follows from the equations of motion (9) that the force density $f_{i}$ acting on a matter distribution $\sigma$ momentarily at rest is

$$
f_{i}=-\sigma \frac{\partial c}{\partial x_{i}}
$$


where $i=1,2,3$ so that $x_{1}, x_{2}, x_{3}$ is $x, y, z$. The total force acting on the frame at this initial instant is computed by integrating this force density over all space. If we substitute for the matter density $\sigma$ using the field equation (11), we recover

$$
\int f_{i} d V=-\int \sigma \frac{\partial c}{\partial x_{i}} d V=-\int \frac{\Delta c}{c} \frac{\partial c}{\partial x_{i}} d V \neq 0
$$

where the integral extends over all of three space. This integral does not, in general, vanish, Einstein noted. Thus there is a net force acting on the mass-frame system that seeks to set it into motion.

This, Einstein observed, violates the "principle of equality of action and reaction." Alternatively, we might observe that it violates both energy and momentum conservation, since the mass-frame system spontaneously acquires both. Einstein could not disguise his alarm. He Wrote (p. 453):

We have recovered a very questionable result. It is quite enough to arouse doubt over the admissibility of the entire theory developed here. This result certainly indicates a lacuna that lies deeply in the foundation of both our investigations. For it can hardly work out that another equation other than equation [(10)] can be brought into consideration from the expression $\left(c_{0}+a x\right)$ found for $c$ for a uniformly accelerated system. This [equation] in turn entails equation [(11)] necessarily.

\section{Seeking an Escape}

Einstein's remarks foreshadow that he will have to give up his pair of field equations (10) and (11). However he was not prepared to take this step without resistance. He sought first to preserve them by modifying other parts of his theory.

The first approach was to consider the fact that the massless frame holding the masses is stressed as it prevents the gravitational collapse of the masses it carries. Earlier work in special relativity had shown that stressed bodies can have unexpected energetic properties. For example, if a stressed body is set in motion, there will be an energy associated with the stress that only appears when the body is in motion. ${ }^{16}$ Might there be a gravitational mass associated with the

\footnotetext{
16 For a survey of these results, see Norton $(1992, \S 9)$.
} 
stresses in the frame that somehow preserves the equality of action and reaction? Einstein explored the possibility by considering a mirrored box that contained radiation; and another box containing an ideal gas. In both cases, the walls of the boxes would become stressed in virtue of the pressures exerted on them by the radiation and the gas. However, Einstein concluded, one could not attribute a gravitational mass to the stressed walls. The gravitational mass of the entire system must be determined solely by its total energy. For only then is the equality of inertial and gravitational mass retained. This equality would be violated if an additional gravitational mass were attributed to the stresses in the box walls. ${ }^{17}$

In the second approach, Einstein considered modifying the theory's expressions for the momentum of a moving mass and for the gravitational gravitation force by multiplying each by some power in $c$, the first by $c^{\alpha}$ and the second by $c^{\beta}$. Einstein briefly recounts his explorations that showed that these modifications precluded a serviceable dynamics.

\section{Modifying the Gravitational Field Equation}

Einstein now bowed to the inevitable. The equality of action and reaction could only be preserved, he concluded (p.455), if his field equations (10) and (11) were modified. We can understand the modification Einstein introduced by reflecting on how ordinary Newtonian gravitation theory and Coulomb electrostatics avoid the problem.

The force density $f_{i}$ on a charge distribution $\rho$ due to the Coulomb potential $\varphi$ is given by

$$
f_{i}=-\rho \frac{\partial \varphi}{\partial x_{i}}
$$

The potential is governed by Poisson's equation

17 Einstein soon returned to the possibility of associating a gravitational mass with stresses in Einstein and Grossmann $(1913, \S I .7)$ through the use of the trace $T$ of the stress-energy tensor as the source density in a scalar field equation. Implementing this choice in Nordström's Lorentz covariant gravitation theory led Einstein to a version of the theory that was only conformally flat. It was, as reported in Einstein and Fokker (1914), governed by a field equation $R=\kappa T$ where $R$ is the curvature scalar and $\kappa$ a constant. For further details, see Norton (1992). 


$$
\Delta \varphi=\left(\frac{\partial^{2}}{\partial x^{2}}+\frac{\partial^{2}}{\partial y^{2}}+\frac{\partial^{2}}{\partial z^{2}}\right) \varphi=\sum_{i=1}^{3} \frac{\partial^{2} \varphi}{\partial x_{i}^{2}}=-k \rho
$$

Proceeding as before, we express the force density $f_{i}$ solely in terms of the potential $\varphi$ by substituting Poisson's equation (17) into (16):

$$
f_{i}=-\rho \frac{\partial \varphi}{\partial x_{i}}=\frac{1}{k}\left(\sum_{m=1}^{3} \frac{\partial^{2} \varphi}{\partial x_{m}^{2}}\right) \frac{\partial \varphi}{\partial x_{i}}=\frac{1}{k} \sum_{m=1}^{3} \frac{\partial}{\partial x_{m}}\left(\frac{\partial \varphi}{\partial x_{m}} \frac{\partial \varphi}{\partial x_{i}}-\frac{1}{2} \delta_{i m}\left(\sum_{n=1}^{3} \frac{\partial \varphi}{\partial x_{n}} \frac{\partial \varphi}{\partial x_{n}}\right)\right)=\frac{1}{k} \sum_{m=1}^{3} \frac{\partial t_{i m}}{\partial x_{m}}
$$

What will prove the most important step in this computation is the third equality. It is merely the computation of an identity in $\varphi$ :

$$
\left(\sum_{m=1}^{3} \frac{\partial^{2} \varphi}{\partial x_{m}^{2}}\right) \frac{\partial \varphi}{\partial x_{i}}=\sum_{m=1}^{3} \frac{\partial}{\partial x_{m}}\left(\frac{\partial \varphi}{\partial x_{m}} \frac{\partial \varphi}{\partial x_{i}}-\frac{1}{2} \delta_{i m}\left(\sum_{n=1}^{3} \frac{\partial \varphi}{\partial x_{n}} \frac{\partial \varphi}{\partial x_{n}}\right)\right)
$$

The last term in the scope of the divergence operator is the Maxwell stress tensor for the Coulomb field, which is defined as

$$
t_{i m}=\frac{\partial \varphi}{\partial x_{m}} \frac{\partial \varphi}{\partial x_{i}}-\frac{1}{2} \delta_{i m}\left(\sum_{n=1}^{3} \frac{\partial \varphi}{\partial x_{n}} \frac{\partial \varphi}{\partial x_{n}}\right)
$$

Equation (18) shows that the force density $f_{i}$ equals the divergence of the stress tensor $t_{i m}$. This fact, we can see, preserves the equality of action and reaction in systems of the type Einstein considered. Take a finite system of charges attached to a rigid frame in a field $\varphi$ whose spatial derivatives $\partial c / \partial x_{i}$ approach zero as we approach spatial infinity. Using a standard computation routinely employed in field theories, Gauss' theorem allows us to compute the $i$-th component of the net force on system of charges $F_{i}$ through

$$
F_{i}=\int_{V} \sum_{k=1}^{3} \frac{\partial t_{i k}}{\partial x_{k}} d v=\int_{A} \sum_{k=1}^{3} t_{i k} n_{k} d a
$$

The first volume integral extends over a volume of space $V$ sufficiently large for it to contain all the charges and such that the first derivatives of the field $\partial \varphi / \partial x_{i}$ are brought arbitrarily close to zero on its surface $A$. The second surface integral extends over the surface $A$ only. The quantity $n_{i}$ is a unit, vector normal to the surface. Since the first derivatives of $\varphi$ can be brought arbitrarily close zero by making $V$ suitably large, the stress tensor $t_{i k}$ can be made arbitrarily small and so 
also $^{18}$ the net force $F_{i}$. This force vanishes if we now take the limit as the volume of integration $V$ exhausts all space. Thus the system of charges and rigid frame experiences no net force. The equality of action and reaction is preserved.

Einstein's gravitational field equation (11) seems so close in form to the Poisson equation for Newtonian gravity (12) and for Coulomb electrostatics (17) that we can easily imagine that some similar computation is possible that would preserve the equality of action and reaction. Einstein's (11) differs only in the addition of field potential term $c$ in the field equation's source term $k c \sigma$. Yet that additional term is enough to overturn the whole calculation. To see why, we merely need to repeat the electrostatic calculation of (18) in Einstein's gravitation theory. Substituting the field equation (11) into the expression (14) for the force on a static mass distribution, we recover:

$$
\begin{aligned}
f_{i} & =-\sigma \frac{\partial c}{\partial x_{i}}=-\frac{1}{k c}\left(\sum_{m=1}^{3} \frac{\partial^{2} c}{\partial x_{m}^{2}}\right) \frac{\partial c}{\partial x_{i}} \\
& =\sum_{m=1}^{3} \frac{\partial}{\partial x_{m}}\left(-\frac{1}{k c}\left(\frac{\partial c}{\partial x_{m}} \frac{\partial c}{\partial x_{i}}-\frac{1}{2} \delta_{i m}\left(\sum_{n=1}^{3} \frac{\partial c}{\partial x_{n}} \frac{\partial c}{\partial x_{n}}\right)\right)-\frac{1}{2 k c^{2}}\left(\sum_{m=1}^{3} \frac{\partial c}{\partial x_{m}} \frac{\partial c}{\partial x_{m}}\right) \frac{\partial c}{\partial x_{i}}\right.
\end{aligned}
$$

where the last equality is an identity. The calculation can almost proceed as before. The force density $f_{i}$ is equal to a divergence, the divergence of a term quadratic in the derivatives of $c$, and a second term. The quantity within the scope of the divergence operator $\sum_{m=1}^{3} \frac{\partial}{\partial x_{m}}(\bullet)$ can be provisionally identified as the gravitational field stress tensor: 19

$$
t_{i m}=-\frac{1}{k c}\left(\frac{\partial c}{\partial x_{m}} \frac{\partial c}{\partial x_{i}}-\frac{1}{2} \delta_{i m}\left(\sum_{n=1}^{3} \frac{\partial c}{\partial x_{n}} \frac{\partial c}{\partial x_{n}}\right)\right)
$$

We are very close to the goal of writing the force density $f_{i}$ as a divergence:

$$
f_{i}=-\sigma \frac{\partial c}{\partial x_{i}}=\sum_{m=1}^{3} \frac{\partial t_{i m}}{\partial x_{m}}
$$

However the second superfluous term of the expression in (22) remains

18 A tacit presumption is that $t_{i k}$ approaches zero faster than the area $A$ grows infinite.

19 Einstein's expression for this stress tensor in (1912b, p. 456) omits the leading minus sign. I believe this is a typographical error in Einstein's paper. 


$$
-\frac{1}{2 k c^{2}}\left(\sum_{m=1}^{3} \frac{\partial c}{\partial x_{m}} \frac{\partial c}{\partial x_{m}}\right) \frac{\partial c}{\partial x_{i}}
$$

It precludes us writing the force density as a divergence. Its presence leads to the non-vanishing force Einstein reported in equation (15) above.

A short calculation shows that this second, troublesome term can be eliminated if it is absorbed into the gravitational field equation (11). This absorption yields the modified gravitational field equation of the second theory of 1912:

$$
\Delta c=\left(\frac{\partial^{2}}{\partial x^{2}}+\frac{\partial^{2}}{\partial y^{2}}+\frac{\partial^{2}}{\partial z^{2}}\right) c=k\left(c \sigma+\frac{1}{2 k c} \sum_{m=1}^{3} \frac{\partial c}{\partial x_{m}} \frac{\partial c}{\partial x_{m}}\right)
$$

This modified field equation solves the dynamical problem. Using it, the force density $f_{i}$ can be written as the divergence of a tensor, $t_{i m}$ of (23). An argument analogous to that of the electrostatic case shows the equality of action and reaction is preserved. A bonus, possibly unexpected, is that Einstein could show that the additional term in the modified field equation

$$
\frac{1}{2 k c} \sum_{m=1}^{3} \frac{\partial c}{\partial x_{m}} \frac{\partial c}{\partial x_{m}}
$$

is equal to the energy density of the gravitational field. Einstein now had the appealing result that ordinary matter density $\sigma$ and the gravitational field energy contribute equally, in arithmetic summation, as the source of the gravitational field:

$$
\Delta c=k(\text { ordinary matter density }+ \text { energy density of the gravitational field })
$$

With this modification, Einstein's theorizing now moved to non-linear field equations, which would be an enduring feature of his development of general relativity and his subsequent unified field theory.

Einstein identified the peril to his theory quite rapidly, sometime between the writing of the first paper (Einstein 1912a) and the second (Einstein 1912b). How did he find it so quickly? Its presence is obvious once one tries the calculation of equation (22) above. Why would Einstein try such a calculation? A striking juxtaposition may answer this last question. The first two sections of Einstein (1912b) tackle a mundane exercise required by the new theory. Einstein asks how electrodynamics must be modified to remain compatible with the new theory of gravity. Einstein shows that all that is required is the addition of factors of $c$ in several places. He then proceeds to check that the resulting modified theory retains the conservation of energy and the 
conservation of momentum. The first computation involves recovering an expression for the electromagnetic field energy density. The second computation leads Einstein to write a modified expression for the Maxwell stress tensor and to show that the modified expression allows retention of the conservation of momentum. The corresponding computation for the gravitational stress tensor of his new theory is the failure that Einstein proceeds to report and that leads to the need for a modification of his gravitational field equation from (11) to (25).

\section{Conflicting Heuristics}

This modification was not just an ad hoc expedient. As we shall see shortly, it embodies a procedure that Einstein could and would use again. It proved to be an invaluable heuristic. The difficulty for Einstein, however, was that this heuristic contradicted the primary heuristic that had played a dominant role in Einstein's thinking on gravity since 1907: the principle of equivalence.

To see the conflict, take what Einstein (1912b, p. 456) correctly gave as an equivalent form of the modified field equation (25):

$$
\Delta(\sqrt{c})=\frac{k}{2} \sqrt{c} \sigma
$$

For the source free $(\sigma=0)$ case of a unidirectional field such as might be produced by unidirectional acceleration, in analogy with (6), we recover

$$
\sqrt{c}=\sqrt{c_{0}}+a x
$$

The difficulty is that uniform acceleration in special relativity produces (6) and not (28). That is, the gravitational field of the principle of equivalence, produced by uniform acceleration, is not a gravitational field admitted by the modified field equation $(25) /(27)$.

One might wonder if there is some scope for modifying the transformation equations (1) used to produce the field represented by (6). Using later ideas, we can see that this is not possible, unless Einstein is prepared to make much more sweeping changes to this theory. That is, if we assume that the spacetime geometry is given by the line element (8), it is shown in Appendix A, equation (42), that the function $c(x, y, z)$ in (8) can vary at most linearly with the spatial coordinates $x, y$ and $z$.

Einstein (1912b, pp. 455-56) reported his reluctance to adopt the modified field equation: 
Therefore I decided with difficulty to take this step, since with it the foundation of the unconditional principle of equivalence is lost. It appears that the latter can only be retained for infinitely small fields.

Presumably the restriction is to infinitely small intervals of space in the direction of the $x$ coordinate. For then the non-linear dependency of $c$ on $x$ in (28) can be approximated in the infinitely small interval for the linear dependency of (6).

All was not lost entirely, Einstein continued. For his derivation of the equations of motion (9) and the modification to the equations of electrodynamics from the principle required only that his transformation equations (2) can be applied to infinitely small spaces. He suggested that the transformation equation (2) be replaced by the more general equations:

$$
\tau=c t \quad \xi=x+\frac{1}{2} c \frac{\partial c}{\partial x} t^{2} \quad \eta=y \quad \zeta=z
$$

where $c$ is an arbitrary function of $x$.

While the outcome was clearly painful for Einstein, there is an unmistakable conclusion concerning Einstein's heuristics. Einstein's first tier and most visible heuristic of the principle of equivalence conflicted with the less visible, second tier heuristic of momentum conservation. The second tier heuristic wins. It is, in the end, a more powerful guide that cannot be overruled.

\section{A Method Reused: The Derivation of the "Entwurf" Field Equations}

The procedure Einstein used in 1912 to correct his gravitational field equation was not something merely to be used once. It could reused in different contexts. That is, for Einstein, it was a method. We know this because he goes to some pains to tell us. The word "method" is his, as we shall see below.

In their "Entwurf" paper of 1913, Einstein and his collaborator, his mathematician friend Marcel Grossmann, published an almost complete version of the general theory of relativity. (Einstein and Grossmann, 1913) What was missing were the now celebrated Einstein gravitational field equations. ${ }^{20}$ In their place, Einstein offered field equations of limited covariance. In his physical part of their joint paper, Einstein addressed the problem of identifying

20 The story of their rejection of generally covariant field equations has been told in abundance elsewhere. See Stachel (1989), Norton (1984) and Renn (2007). 
these equations. Following a now familiar approach, he posited that these gravitational field equations would have the form

$$
\Gamma^{\mu \nu}=k T^{\mu \nu}
$$

where $T^{\mu v}$ is the stress-energy tensor for ordinary matter and $k$ is a constant. The gravitation tensor, $\Gamma^{\mu v}$, is a quantity constructed from the metric tensor $g_{\mu v}$ and its first and second coordinate derivatives. Unlike his later theory, this tensor was permitted limited covariance. In the case of a spacetime whose metric differed only in small quantities from that of a Minkowski spacetime, Einstein specified (pp. 13-15) that the gravitation tensor would have the form (in more modern notation):

$$
\Gamma^{\mu v}=\frac{\partial}{\partial x^{\alpha}}\left(g^{\alpha \beta} \frac{\partial g^{\mu v}}{\partial x^{\beta}}\right)+\begin{gathered}
\text { further terms that vanish in the } \\
\text { formation of the first approximation }
\end{gathered}
$$

How could these further terms be found?

Einstein saw that his situation was quite similar to that of 1912. One could conceive his first gravitational field equation (11) merely as an approximation to the correct equation, merely lacking the higher order terms introduced in the second gravitational field equation (25). Einstein had found these higher order terms by requiring that substitution of the force density equation (16) into the field equation must produce an identity from which the conservation of momentum could be recovered. Without mentioning the embarrassing retraction of 1912, Einstein now sought to employ the same method in his new "Entwurf" theory. He was concerned to convey clearly to the reader the method that would be used. To do so, he recapitulated the analysis given above in Section 10 for the familiar case of electrostatics. ${ }^{21}$ I quote him at length (p. 14):

The momentum energy law will serve us in the discovery of these terms. So that the method used is clearly delineated, I now want to apply it to a generally known example.

21 A curious omission is that Einstein never states the key point explicitly: that conservation of momentum is assured by the existence of the Maxwell stress tensor. Perhaps he assumed it would be obvious to the reader? 
In electrostatics, $-\frac{\partial \varphi}{\partial x_{v}} \rho$ is the $v^{\text {th }}$ component of the momentum per unit volume imparted to matter, in case $\varphi$ signifies the electrostatic potential, $\rho$ the electric [charge] density. A differential equation is sought for $\varphi$ of such a kind that the momentum law is always satisfied. It is well-known that the equation

$$
\sum_{v} \frac{\partial^{2} \varphi}{\partial x_{v}^{2}}=\rho
$$

solves the exercise. That the momentum law is satisfied follows from the identity

$$
\sum_{\mu} \frac{\partial}{\partial x_{\mu}}\left(\frac{\partial \varphi}{\partial x_{v}} \frac{\partial \varphi}{\partial x_{\mu}}\right)-\frac{\partial}{\partial x_{v}}\left(\frac{1}{2} \sum_{\mu}\left(\frac{\partial \varphi}{\partial x_{\mu}}\right)^{2}\right)=\frac{\partial \varphi}{\partial x_{v}} \sum_{\mu} \frac{\partial^{2} \varphi}{\partial x_{\mu}^{2}}\left(=-\frac{\partial \varphi}{\partial x_{v}} \cdot \rho\right)
$$

Therefore if the momentum law is satisfied, for each $v$ an identical equation of the following construction must exist: on the right hand side is $-\frac{\partial \varphi}{\partial x_{v}}$ multiplied by the by the left hand side of the differential equation. On the left hand side of the identity is a sum of differential quotients.

If the differential equation for $\varphi$ were not yet known, then the problem of its discovery may be reduced to that of the discovery of this identical equation. What is essential for us now is the knowledge that this identity may be derived if one of the terms appearing in it is known. [Einstein's emphasis] One has nothing more to do than to apply repeatedly the rule for differentiation of a product in the form

$$
\frac{\partial}{\partial x_{v}}(u v)=\frac{\partial u}{\partial x_{v}} v+\frac{\partial v}{\partial x_{v}} u
$$

and

$$
u \frac{\partial v}{\partial x_{v}}=\frac{\partial}{\partial x_{v}}(u v)-\frac{\partial u}{\partial x_{v}} v
$$

and finally to place terms that are differential quotients on the left hand side and the remaining [terms] on the right hand side. If one proceeds, f[or] e[xample] from the first term of the above identity, one obtains the sequence 


$$
\begin{aligned}
\sum_{\mu} \frac{\partial}{\partial x_{\mu}}\left(\frac{\partial \varphi}{\partial x_{v}} \frac{\partial \varphi}{\partial x_{\mu}}\right) & =\sum_{\mu} \frac{\partial \varphi}{\partial x_{v}} \cdot \frac{\partial^{2} \varphi}{\partial x_{\mu}^{2}}+\sum_{\mu} \frac{\partial \varphi}{\partial x_{v}} \cdot \frac{\partial^{2} \varphi}{\partial x_{v} \partial x_{\mu}} \\
& =\frac{\partial \varphi}{\partial x_{v}} \cdot \sum_{\mu} \frac{\partial^{2} \varphi}{\partial x_{\mu}^{2}}+\frac{\partial}{\partial x_{v}}\left\{\frac{1}{2} \sum_{\mu}\left(\frac{\partial \varphi}{\partial x_{\mu}}\right)^{2}\right\}
\end{aligned}
$$

from which the above identity follows through rearrangement.

Einstein now proceeded to use this method to derive the gravitational field equations of his "Entwurf" theory. The derivation was essentially just the derivation of the second gravitational field equation of 1912, but now promoted to the more complicated context of the "Entwurf" theory. In place of the single gravitational potential $c$ was the multi-component metric tensor $g_{\mu \nu}$. In place of momentum conservation and the Maxwell stress tensor was the requirement of conservation of energy-momentum and the stress-energy tensor of the gravitational field.

The resulting gravitation tensor $\Gamma^{\mu \nu}$ is given in the Appendix below as equation (43). The promoted computations are considerably more complicated than those of the 1912 theory. Grossmann's (1913, pp. 37-38) part contains the derivation of the essential identity, which covers two journal pages. The details of these formulae are unilluminating for our present interests and I will spare the reader parading them.

\section{Conflicting Heuristics Again}

While the promotion of the method of 1912 had now provided Einstein with a unique set of gravitational field equations for his new "Entwurf" theory, the conflict of heuristics present in 1912 remained and in a more damaging form. The principle of equivalence had assured Einstein that uniform acceleration produces a homogeneous gravitational field. We saw above that Einstein's modified field equation of 1912 no longer identified this acceleration field in its totality as a gravitational field. The best Einstein could say was that infinitely small parts of the field were identified individually as a gravitational field. In the "Entwurf" theory, this last slender thread to the principle was broken. For the only static spacetimes with the line element (8) allowed by the source free "Entwurf" gravitational field equations were those with $c=$ constant. (See Appendix B. Equation (46).) That is merely the spacetime of special relativity, Minkowski spacetime. 
The immediate problem was that Einstein could not present the "Entwurf" theory as realizing the idea implicit in the principle of equivalence. For in this new theory, uniform acceleration did not produce a gravitational field that was recognized by the theory's gravitational field equations. Hence, as noted already in Norton $(1985, \S 4.3)$, during the time of the "Entwurf" theory, Einstein tended to avoid detailed discussion of the principle of equivalence.

In his part of Einstein and Grossmann (1913), the principle (“Äquivalenz-Hypothese”) is introduced (p.3) with the restriction to homogeneous gravitational fields of infinitely small extension. It is recalled subsequently $(\S 1)$ only as the basis of the 1912 theory, which is summarized briefly. In his later Einstein (1913, pp. 1254-55), the principle is presented as a vividly developed thought experiment concerning physicists who awaken from a drugged sleep in a closed, accelerating box. Einstein does not, however, develop the specific results such as the line element (4) above. Soon after, Einstein and Grossmann (1914, p. 216) reaffirm

The whole theory proceeds from the conviction that all physical processes in a gravitational field play out in exactly the same way as the corresponding processes play out without a gravitational field, in case one relates them to an appropriately accelerated (three dimensional) coordinate system. (“Äquivalenzhypothese”)

It is notable that Einstein and Grossman leave open just what form the "appropriate" acceleration can take. They fail to specify the uniform acceleration and homogeneous gravitational fields of Einstein's earlier formulations and those of his writings after 1915. In November 1914, Einstein (1914) published a definitive review article on the latest form of his theory. The principle of equivalence is now absent in name from the introductory discussion. Instead, Einstein reflects on rotational motion and urges (p. 1032) that the centrifugal field appearing in a rotating frame of reference should be conceived as a gravitational field. ${ }^{22}$

After November 1915, when Einstein had finally secured a generally covariant theory, he could once again conceive of the field of uniform acceleration as gravitational. The principle of equivalence was restored in its original form to its original prominence in Einstein's accounts of

22 At this time, as Janssen (1999) recounts in some detail, through a calculation error, Einstein had convinced himself that this centrifugal field is a solution of the "Entwurf" gravitational field equations. 
his theory. It appears in the introductory discussion $(\S 2)$ of his new review article (Einstein 1916); and in Ch.XX of Einstein's (1920b) popular book on relativity, whose preface is dated December 1916.

The hiatus in discussion of the principle of equivalence coincides with the time of the "Entwurf" theory. Thus is it natural to suppose that Einstein knew that the original principle failed completely in his theory. Unfortunately Einstein never explicitly acknowledged the failure. What complicates the problem is that some of Einstein's narratives (cited above) still include it. What deepens the problem is that Einstein repeatedly employed a spacetime with a line element (8) to represent the gravitational field outside a spherically symmetric body, such as was assumed for the sun. The difficulty is that this field must conform with the source free gravitation field equations and, as shown in Appendix B, these equations admit nothing but a flat Minkowski spacetime for a spacetime with this line element.

While Einstein's silence makes it impossible for us to be certain, I think it most plausible that Einstein knew of the problem but found it expedient to remain silent about it. For once a successful theory has been achieved, what could be gained by announcing incompatibilities between the theory and the specifics of the ideas that led to it? If uniform acceleration does not produce a gravitational field in the theory, then other accelerations might; and Einstein mistakenly believed this to be the case for rotation. As to the applicability of the line element (8) to the spacetime surrounding the sun, it is notable that Einstein's derivations all employ approximations. ${ }^{23}$ Thus the negative result of Appendix B below could be avoided if the line element of these spacetimes had the form (8) only approximately, that is, to the order of the approximation of his calculations. I find it most plausible that this was Einstein's view.

There is evidence that Einstein knew that static gravitational fields, such as that of the sun, admitted deviations from spatial flatness that were non-zero in the second order of smallness. The most direct evidence comes in a draft manuscript of calculations co-authored by Einstein and his friend Michele Besso, mostly in mid 1913. (Klein et al., 1995, Doc. 14) They compute

23 See for example Einstein $(1913, \S 8)$ and Einstein $(1914, \S 17)$. In a letter of March 19, 1915 (Schulman et al., 1998, Doc. 63.), Einstein sought to reassure Erwin Freundlich that the spacetime surrounding the sun has the metric associated with (8)/ (36). Einstein presented a short proof that demonstrates the result only in low order approximation. 
the gravitational field of the sun to second order in the "Entwurf " theory and, on a page in Einstein's hand, non-zero second order deviations are recovered. ${ }^{24}$

What is regrettable is that Einstein does not directly affirm these deviations in his publications from the time. Einstein and Grossmann (1913, p. 7) presents the line element (8) (in the equivalent form of the tensor (36)) as applying to static gravitational fields "of the previously considered type." This presumably refers to those of the earlier 1912 theory. If Einstein intended the remark not to apply to the present "Entwurf" theory as well, only the most perspicacious of readers could have realized it.

In November 1915, after Einstein had returned to generally covariant gravitational field equations, the error was discovered in the context of Einstein's successful explanation of the anomalous motion of Mercury. He then remarked (Einstein 1915b, p. 834) on the surprising 25 appearance of non-constant components like $\mathrm{g}_{11}, \mathrm{~g}_{22}$ and $\mathrm{g}_{33}$ in the metric field of the sun: "the [non-constancies of the] components $\mathrm{g}_{11}$ to $\mathrm{g}_{33}$ differ from zero already in magnitudes of the first order. [my emphasis]" This emphasized phrase might not be needed, unless Einstein already had expected such deviations only to be of higher order.

\section{The Method Lives On}

After the "Entwurf" paper, the essential ideas behind the method of generating field equations did not disappear, but merely receded. They were absorbed into Einstein's analyses and, while no longer explicitly delineated, continued to exercise a controlling influence on his theorizing.

The gravitational field equations of the "Entwurf" theory were not generally covariant. The pressing problem for Einstein in 1913 and 1914 was to determine the extent of his new theory's covariance. The ideas behind the method of 1912 and 1913 now became the vehicle for

24 See Equations 40 and 42 in (Klein et al., 1995, p. 370) and the associated editorial discussion on p. 349.

25 The word surprise is Einstein's from a letter to Michele Besso of December 10, 1915: "You will be surprised by the appearance of the $g_{11} \ldots g_{33}$." (Schulmann et al, 1998, Doc. 162, p. 218). 
determining this extent. To this end, Einstein and Grossman (1914, p. 217) wrote the "Entwurf" field equations (in modernized notation) as:

$$
\frac{\partial}{\partial x^{\alpha}}\left(\sqrt{-g} g^{\alpha \beta} g_{\sigma \mu} \frac{\partial g^{\mu v}}{\partial x^{\beta}}\right)=\kappa \sqrt{-g}\left(T_{\sigma}^{v}+t_{\sigma}^{v}\right)
$$

where $t_{\sigma}{ }^{v}$ is an expression quadratic in first derivatives of the metric tensor and identified as the stress-energy tensor of the gravitational field. The conservation of energy and momentum was written as

$$
\frac{\partial}{\partial x^{v}}\left(\sqrt{-g}\left(T_{\sigma}^{v}+t_{\sigma}^{v}\right)\right)=0
$$

Following the earlier method, we should expect that substituting (32) into (33) yields an identity in the metric tensor $g_{\mu \nu}$. The resulting identity in $g_{\mu \nu}$ is

$$
B_{\sigma}=\frac{\partial^{2}}{\partial x^{v} \partial x^{\alpha}}\left(\sqrt{-g} g^{\alpha \beta} g_{\sigma \mu} \frac{\partial g^{\mu \nu}}{\partial x^{\beta}}\right)=0
$$

This identity took on a new significance. It could only be expected to hold in coordinate systems in which the original equations (32) and (33) held. Einstein and Grossmann could now use the identity as the condition that picks out just those coordinate systems in which the "Entwurf" theory held.

This "adapted coordinate condition," as they called it, became a central feature of the development of the "Entwurf" theory. Einstein and Grossmann (1914) and Einstein (1914) developed a variational formalism for the "Entwurf" theory. A major goal of the formalism was to demonstrate that this adapted coordinate condition did precisely characterize exactly the extent of covariance of the theory and that is was the maximum covariance permitted by Einstein's original interpretation of the hole argument.

When Einstein returned to general covariance and formulated the now familiar generally covariant gravitational equations, the ideas behind this repurposed method and the variational formalism persisted. The major difference was that the identity replacing (34) no longer picked out just those few coordinate systems in which the theory held. For under general covariance, the final theory held in all coordinate system. Thus the replacement identity must hold in all coordinate systems. It was recognized later to be none other that the contracted Bianchi identity:

$$
\left(R^{\mu v}-\frac{1}{2} g^{\mu v} R\right)_{; v}=0
$$


where $R^{\mu \nu}$ is the Ricci tensor.

Einstein's original method of 1912 and 1913 now survives as the most familiar means of arriving at the gravitational field equations of general relativity. It proceeds by arguing, as did Einstein (1923, pp. 92-93), that the gravitational field equations have the form

$$
G^{\mu \nu}=\kappa T^{\mu \nu}
$$

The generally covariant gravitation tensor $G^{\mu \nu}$ is formed from the metric tensor and its first and second derivatives; and is linear in the second derivatives. It follows that $G^{\mu \nu}$ must be a linear combination of $R^{\mu \nu}$ and $g^{\mu \nu} \mathrm{R}$. If conservation of energy momentum

$$
T^{\mu \nu}{ }_{; v}=0
$$

is to be maintained, this linear combination must have a vanishing covariant divergence. We read from (35) that the gravitation tensor is what is now called the Einstein tensor:

$$
G^{\mu v}=R^{\mu v}-\frac{1}{2} g^{\mu v} R
$$

\section{Einstein's Two Tier Heuristics}

To return to our starting point, how are we to think of the heuristics that guided Einstein to his general theory of relativity? His starting point in 1907 was the principle of equivalence. There can be no doubt that Einstein held firmly to the idea that this principle was the foundation from which he proceeded, even as the principle delivered results in contradiction with his evolving theory. Here is how Einstein recalled the situation in a letter of September 12, 1950, to Laue. At issue was the fact that the Riemann curvature tensor vanishes in the rotating coordinate system adapted to a rotating disk in Minkowski spacetime. Einstein replied 26

It is true that in that case the $R_{i k l m}$ vanish, so that one could say: "there is no gravitational field present." However, what characterizes the existence of a gravitational field from the empirical standpoint is the non-vanishing of the $\Gamma_{i k} l$ [coefficents of the affine connection], not the non-vanishing of the $R_{i k l m}$. If one does not think intuitively in such a way, one cannot grasp why something like curvature should have anything at all to do with gravitation. In any case, no

26 As quoted in Norton $(1985, \S 11)$. 
reasonable person would have hit upon such a thing. The key for understanding of the equality of inertial and gravitational mass is missing.

In retrospect, we can see the most important idea that the principle of equivalence delivered to Einstein. It was, as is argued in Norton $(1985, \S 12)$, that the Minkowski spacetime of special relativity was not to be conceived as a gravitation free spacetime. Rather gravitation was already present in it as a special case. That gave Einstein the crucial clue that a gravitation theory could be constructed, not by adding a gravitational field to that spacetime, but generalizing the structures already present in Minkowski spacetime.

The difficulty was that the principle of equivalence gave Einstein more than this vital clue. It also delivered gravitational fields to Einstein that contradicted his evolving theories of 1912 and 1913. If the principle of equivalence was inviolable, Einstein would have had to abandon these theories. He did not; the principle proved dispensable. Rather he first reduced the principle of equivalence in 1912 to a weak version that obtained only in the infinitely small and then in 1913 and 1914 to a vaguer guide with an imprecisely circumscribed expression. The principle may have taken pride of place in his overarching conceptions, but it enjoyed no such prominence in the practicalities of his theorizing.

Instead Einstein could proceed with quite definite theories because a second tier of heuristics were still guiding him. In the account above, one has been singled out as having special importance. ${ }^{27}$ It is the idea that the gravitational field equations must conform with energy and momentum conservation. Unlike the principle of equivalence, that demand was inviolable. It provided a method that guided Einstein to quite specific field equations in 1912 and in 1913 and persists in modern presentations of general relativity.

Is this example of a two-tiered structure of heuristics in Einstein's work exceptional? A second, related example has been explored in some detail by Michel Janssen and Jürgen Renn (2007). In November 1915, Einstein (1915a) reported to the Prussian Academy that he had abandoned his "Entwurf" theory. He presented in its place a new theory of near general covariance that would shortly be extended to full general covariance. Einstein made clear that,

27 Another essential requirement was that his new gravitation theory revert to Newtonian gravitation theory in the case of weak, static gravitational fields. 
once he had lost faith in his earlier theory, considerations of covariance were his primary guide: (p. 778)

Thus I came back ${ }^{[28]}$ to the demand of a more general covariance of the field equations, from which I had departed three years ago, when I worked together with my friend Grossmann, only with a heavy heart.

His reflections devolved into a poetic tribute to the mathematical methods associated with general covariance (p. 779)

Hardly anyone who has truly understood it can resist the charm of this theory; it signifies a real triumph of the method of the general differential calculus, founded by Gauss, Riemann, Christoffel, Ricci and Levi-Civita.

Janssen and Renn, however, have pointed out that the theory then presented by Einstein could be produced by making a small adjustment to the variational formulation of the "Entwurf" theory. A derivative of the metric tensor would be replaced by a Christoffel symbol, otherwise leaving the formalism unchanged. The cogency of the ensuing theory was assured by the results of the earlier formulation. In particular, the modified theory would be assured to conform with the conservation of energy and momentum.

There is no reason to doubt that Einstein conveyed accurately his perception of the overriding importance of covariance considerations. That would be a natural way for him to recall his recognition that the modified theory was the same as one of near general covariance, recoverable from the Riemann tensor. However it obscures how powerfully his further demands constrained his choices.

We see in this example a similar double tiered structure of heuristics. Covariance considerations loomed large in Einstein's thinking as the first tier. However they were quite dispensable. Einstein had abandoned them in 1913 with the formulation of the "Entwurf" theory, whose covariance properties were then unclear. Considerations such as the energy momentum conservation and the Newtonian limit, however, were inviolable and formed the second tier that continued to guide and circumscribe his theorizing. In November 1915, Einstein could return to more general covariance precisely because he had in hand a formalism that preserved the demands of this second tier.

28 "So gelangte ich ... zurück..." 


\section{Conclusion}

It is tempting to say that Einstein did not really need the principle of equivalence to guide him to general relativity. The crucial clue that Minkowski spacetime is already gravitational could have been gleaned from a widely known fact, itself brought to prominence by Einstein's work. It is the remarkable equality of inertial and gravitational mass in Newtonian theory. This equality leads to the result that trajectories of bodies in free fall are independent of their mass. They are, in retrospect, tracing out for us the geometry of a curved spacetime associated with gravity. Might that have been enough to guide Einstein or another theorist to general relativity?

Of course, when our concern is the discovery of a theory as exceptional in relation to what went before as general relativity, it is foolhardy to try to imagine how things could have been otherwise. I will not persist. We saw above that Einstein insisted that without the principle of equivalence "no reasonable person" could have found general relativity. However, just as I cannot really know how it would have been if things were otherwise, is not the same true for Einstein?

\section{Appendix A. Computing Spacetime Curvature in Einstein's 1912}

\section{Theory}

Einstein's 1912 theory of static gravitational fields attributed properties to space and time equivalent to spacetimes of his later general theory of relativity with a spacetime metric:

$$
g_{\mu v}=\left[\begin{array}{llll}
-1 & & & \\
& -1 & & \\
& & -1 & \\
& & & c^{2}(x, y, z)
\end{array}\right] \quad g^{\mu v}=\left[\begin{array}{llll}
-1 & & & \\
& -1 & & \\
& & -1 & \\
& & & 1 / c^{2}(x, y, z)
\end{array}\right]
$$

where the spacetime coordinates are $(x, y, z, t)=\left(x^{1}, x^{2}, x^{3}, x^{4}\right)$ and Greek indices $\mu, v$ take values 1,2,3 and 4. In (36), $c$ is a function of $x, y$ and $z$, but not $t$. Following the notational conventions of Einstein (1923, p. 79), we write the coefficients of the connection as

$$
\Gamma_{\mu \nu}^{\alpha}=\frac{1}{2} g^{\sigma \alpha}\left(\frac{\partial g_{\mu \alpha}}{\partial x^{v}}+\frac{\partial g_{v \alpha}}{\partial x^{\mu}}-\frac{\partial g_{\mu \nu}}{\partial x^{\alpha}}\right)
$$

where summation over repeated indices is implied. Substituting (36) in (37), the only non-zero terms are 


$$
\Gamma_{44}^{i}=c \frac{\partial c}{\partial x^{i}} \quad \Gamma_{i 4}^{4}=\Gamma_{4 i}^{4}=\frac{1}{c} \frac{\partial c}{\partial x^{i}}
$$

where a Latin index $i=1,2,3$, is used to identify the spatial coordinates $(x, y, z)=\left(x^{1}, x^{2}, x^{3}\right)$. The Ricci tensor, as given by Einstein (1923, p. 85), is

$$
R_{\mu \nu}=-\frac{\partial \Gamma_{\mu v}^{\alpha}}{\partial x^{\alpha}}+\frac{\partial \Gamma_{\mu \alpha}^{\alpha}}{\partial x^{v}}+\Gamma_{\mu \beta}^{\alpha} \Gamma_{v \alpha}^{\beta}-\Gamma_{\mu \nu}^{\alpha} \Gamma_{\alpha \beta}^{\beta}
$$

Using the values of (38), after some calculations, the Ricci tensor reduces to 29

$$
\begin{aligned}
& R_{44}=-c \Delta c=-c\left(\frac{\partial^{2}}{\partial x^{2}}+\frac{\partial^{2}}{\partial y^{2}}+\frac{\partial^{2}}{\partial z^{2}}\right) c \\
& R_{i k}=\frac{1}{c} \frac{\partial^{2} c}{\partial x^{i} \partial x^{k}}, \text { so that } R_{11}=\frac{1}{c} \frac{\partial^{2} c}{\partial x^{2}}, R_{12}=\frac{1}{c} \frac{\partial^{2} c}{\partial x \partial y}, \text { etc } \\
& R_{i 4}=R_{4 i}=0
\end{aligned}
$$

Finally, the Riemann curvature scalar is

$$
R=g^{\mu v} R_{\mu v}=\frac{1}{c^{2}} R_{44}-R_{11}-R_{22}-R_{33}=-\frac{2}{c} \Delta c
$$

Einstein's source free gravitational field equations of $1915, R_{\mu v}=0$, lead to highly restricted results. The spatial part, $R_{i k}=0$, alone is sufficient to ensure that $c$ depends at most linearly on the spatial coordinates $x, y$ and $z$. That is

$$
c(x, y, z)=A+B x+C y+D z
$$

where $A, B, C$ and $D$ are constants. Equation (42) also applies to the special case of flat spacetime, when the Riemann curvature tensor vanishes. For in that case, its first contraction must also vanish, $R_{\mu \nu}=0$.

Had Einstein set his source density in his field equation (11), $\Delta c=k c \sigma$, equal to the trace of the stress-energy tensor of ordinary matter, that is, $\sigma=T$, then it follows from (41) that the field equation (11) would be equivalent to

$$
-R=(2 / c) \Delta c=2 k \sigma=2 k T
$$

29 These formulae are accurate to all orders. They differ from Stachel's (1989, p. 67) formulae, which are computed only, in Stachel's expression, in "linearized approximation." 


\section{Appendix B. Computing the Gravitation Tensor of the Einstein- Grossmann Theory for a Static Gravitational Field}

The gravitation tensor of limited covariance of Einstein and Grossmann (1913,p. 15) is given in more modern notation as:

$$
\begin{aligned}
\Gamma^{\mu v}=\frac{1}{\sqrt{-g}} \frac{\partial}{\partial x^{\alpha}} & \left(\sqrt{-g} g^{\alpha \beta} \frac{\partial g^{\mu v}}{\partial x^{\beta}}\right)-g^{\alpha \beta} g_{\tau \sigma} \frac{\partial g^{\mu \tau}}{\partial x^{\alpha}} \frac{\partial g^{v \rho}}{\partial x^{\beta}} \\
& +\frac{1}{2} g^{\alpha \mu} g^{\beta v} \frac{\partial g_{\tau \rho}}{\partial x^{\alpha}} \frac{\partial g^{\tau \rho}}{\partial x^{\beta}}-\frac{1}{4} g^{\mu v} g^{\alpha \beta} \frac{\partial g_{\tau \rho}}{\partial x^{\alpha}} \frac{\partial g^{\tau \rho}}{\partial x^{\beta}}
\end{aligned}
$$

Evaluating this tensor for the static spacetimes (36), as conceived in the Einstein and Grossmann theory, we find the only non-zero derivatives of the metric tensor are:

$$
\frac{\partial g_{44}}{\partial x^{i}}=2 c \frac{\partial c}{\partial x^{i}} \quad \frac{\partial g^{44}}{\partial x^{i}}=-\frac{2}{c^{3}} \frac{\partial c}{\partial x^{i}}
$$

where $i=1,2,3$. After some straightforward computations, we recover

$$
\begin{gathered}
\Gamma^{44}=\frac{2}{c^{3}} \sum_{i=1}^{3} \frac{\partial^{2} c}{\partial\left(x^{i}\right)^{2}}-\frac{1}{c^{4}} \sum_{i=1}^{3}\left(\frac{\partial c}{\partial x^{i}}\right)^{2} \quad \Gamma^{i i}=-\frac{2}{c^{2}}\left(\frac{\partial c}{\partial x^{i}}\right)^{2}+\frac{1}{c^{2}} \sum_{i=1}^{3}\left(\frac{\partial c}{\partial x^{i}}\right)^{2} \\
\Gamma_{(i \neq k)}^{i k}=-\frac{2}{c^{2}} \frac{\partial c}{\partial x^{i}} \frac{\partial c}{\partial x^{k}} \quad \Gamma^{i 4}=\Gamma^{4 i}=0
\end{gathered}
$$

where all summations are explicit. No summations are implied. For the source free case, the gravitational field equations of the theory are $\Gamma^{\mu v}=0$. The component equation $\Gamma^{44}=0$

corresponds to the source free form of the second field equation (25) of Einstein's second theory of 1912. The remaining component equations, however, have terms in the first derivatives $\partial c / \partial x^{i}$ only. The three equations $\Gamma^{i i}=0$, for $i=1,2,3$. Are sufficient to force $\partial c / \partial \mathrm{x}^{i}=0$ for $i=1,2,3$. That is, we must have

$$
c(x, y, z)=\text { constant }
$$

\section{References}

Einstein, Albert (1907) “Das Relativitätsprinzip und die aus demselben gezogenen Folgerungen,” Jahrbuch der Radioaktivität und Electronik, 4, pp. 411-462.

Einstein. Albert (1911) "Über den Einfluss des Schwerkraft auf die Ausbreitung des Lichtes," Annalen der Physik 35, pp. 898-908. 
Einstein, Albert (1912a) "Lichtgeschwindigkeit und Statik des Gravitationsfeldes," Annalen der Physik, 38, pp. 355-69.

Einstein, Albert (1912b) “Zur Theorie des statischen Gravitationsfeldes," Annalen der Physik, 38, pp. 443-58.

Einstein, Albert (1913) “Zum gegenwärtigen Stande des Gravitationsproblems," Physikalische Zeitschrift 14, pp. 1249-1262.

Einstein, Albert (1914) "Die formale Grundlage der allgemeinen Relativiätstheorie," Königlich Preussische Akademie des Wissenschaften (Berlin), 1914, pp. 1013-1085.

Einstein, Albert (1915a) “Zur allgemeinen Relativitätstheorie,” Königlich Preussische Akademie des Wissenschaften (Berlin), 1915, pp. 778-85.

Einstein, Albert (1915b) "Erklärung der Perihelbewegung des Merkur aus der allgemeinen Relativitätstheorie,” Königlich Preussische Akademie des Wissenschaften (Berlin), 1915, pp. 831-39.

Einstein, Albert (1916) "Die Grundlage der allgemeinen Relativitätstheorie," Annalen der Physik, 49, pp. 769-822.

Einstein, Albert (1920a) "Grundgedanken und Methoden der Relativitätstheorie, in ihrer Entwicklung dargestellt," Doc. 31 in, M. Janssen et al. eds, The Collected Papers of Albert Einstein. Volume 7. Princeton: Princeton University Press, 2002.

Einstein, Albert (1920b) Relativity: The Special and the General Theory. 3rd ed. London: Methuen.

Einstein, Albert (1923) The Meaning of Relativity. Princeton: Princeton University Press.

Einstein, Albert (1933) "Notes on the Origin of the General Theory of Relativity." In Ideas and Opinions, pp. 285-290. Translated by Sonja Bargmann. New York: Crown, 1954.

Einstein, Albert and Grossmann, Marcel (1913) Entwurf einer Verallgemeinerten Relativitätstheorie und einer Theorie der Gravitation. Leipzig u. Berlin: B. G. Teubner.

Einstein, Albert and Grossmann, Marcel (1914) "Kovarianzeigenschaften der Feldgleichungen der auf die verallgemeinerte Relativitätstheorie gegründeten Gravitationstheorie," Zeitschift für Mathematik und Physik, 63, pp. 215-25.

Einstein, Albert and Rosen, Nathan (1935) "The Particle Problem in the General Theory of Relativity," Physical Review, 48, pp. 73-77. 
Janssen, Michel (1999) "Rotation as the Nemesis of Einstein's Entwurf Theory," pp. 127-157 in Hubert Goenner et al . (eds.), The Expanding Worlds of General Relativity. Einstein Studies. Vol. 7 Boston: Birkhäuser.

Janssen, Michel (2014) “'No Success Like Failure ...’: Einstein’s Quest for General Relativity, 1907-1920.” pp. 167-227 in Michel Janssen and Christoph Lehner, eds. The Cambridge Companion to Einstein. Cambridge: Cambridge University Press,.

Janssen, Michel and Renn, Jürgen (2006) "Untying the Knot: How Einstein Found hisWay Back to the Field Equations Discarded in the Zürich Notebook," pp. 839-925 in Jürgen Renn (ed.). The Genesis of General Relativity, Vol. 2 Einstein's Zurich Notebook: Commentary and Essays. Dordrecht: Springer.

Klein, Martin J.; Kox, A. J.; Renn, Jürgen; and Schulman, Robert, eds., (1995) Collected Papers of Albert Einstein. Volume 4. The Swiss Years: Writings 1912-1914. Princeton: Princeton University Press.

Lorentz, Hendrik A. (1920) The Einstein Theory of Relativity: A Concise Statement. New York: Brentano's publishers.

Norton, John D. (1984) “How Einstein Found His Field Equations: 1912-1915,” Historical Studies in the Physical Sciences, 14, pp. 253-315.

Norton, John D. (1985) “What was Einstein's Principle of Equivalence?" Studies in History and Philosophy of Science, 16 (1985), pp. 203-246; reprinted in D. Howard and J. Stachel (eds.), Einstein and the History of General Relativity: Einstein Studies Vol. 1, Boston: Birkhauser, 1989, pp. 5-47.

Norton, John D. (1992) "Einstein, Nordström and the early Demise of Lorentz-covariant, Scalar Theories of Gravitation," Archive for History of Exact Sciences, 45, pp.17-94.

Norton, John D. (1993) “General Covariance and the Foundations of General Relativity: Eight Decades of Dispute," Reports on Progress in Physics, 56, pp.791-858.

Norton, John D. (1995) "Mach's Principle before Einstein." pp.9-57 in J. Barbour and H. Pfister, eds., Mach's Principle: From Newton's Bucket to Quantum Gravity: Einstein Studies, Vol. 6. Boston: Birkhäuser.

Norton, John D. (2004) “Einstein's Investigations of Galilean Covariant Electrodynamics prior to 1905," Archive for History of Exact Sciences, 59, pp. 45-105. 
Norton, John D. (2006) “Atoms Entropy Quanta: Einstein's Miraculous Argument of 1905,” Studies in History and Philosophy of Modern Physics, 37, pp. 71-100.

Renn, Jürgen (2007) The Genesis of General Relativity. Einstein's Zurich Notebook:

Introduction and Source. Volume 1. Einstein's Zurich Notebook: Commentary and Essays. Volume 2. Boston Studies in the Philosophy of Science. Dordrecht: Springer.

Schulmann, Robert; Kox, A. J.; Janssen, Michel and Illy, József, eds., (1998) The Collected Papers of Albert Einstein. Volume 8, Part A. The Berlin Years: Correspondence 19141917. Princeton: Princeton University Press.

Stachel, John (1989) “Einstein's Search for General Covariance, 1912-1915,” pp. 63-100 in Don Howard and John Stachel, eds., Einstein and the History of General Relativity. Boston: Birkhäuser. 